Proyecciones

Vol. $11 \mathrm{~N}^{\circ}$ 2, pp.125-129 Diciembre 1992

Universidad Católica del Norte

Antofagasta - Chile

\title{
PERFECT MEASURES AND THE DUNFORD-PETTIS PROPERTY*
}

JOSE AGUAYO G. and JOSE SANCHEZ H.

Universidad de Concepción, Concepción, Chile

\begin{abstract}
Let $X$ be a completely regular Hausdorff space. We denote by $C_{b}(X)$ the Banach space of all real-valued bounded continuous function's on $X$ endowed with the supremum-norm. $M_{p}(X)$ denotes the subspace of the $\left(C_{b}(X),\|\|\right)^{\prime}$ of all perfect measures on $X$ and $\beta_{p}$ denotes a topology on $C_{b}(X)$ whose dual is $M_{p}(X)$.

In this paper we give a characterization of $\mathrm{E}$-valued weakly compact operators which are $\beta$-continuous on $C_{b}(X)$, where $E$ denotes a Banach space. We also prove that $\left(C_{b}(X), \beta_{\mathrm{p}}\right)$ has strict Dunford-Pettis property and, if $X$ contains a $\sigma$-compact dense subset, $\left(C_{b}(X), \beta_{p}\right)$ has Dunford-Pettis property.
\end{abstract}

* 1991 Mathematics subject classification. Primary 46E10, 46G10. Secondary $47 \mathrm{~B} 38$.

Key words and phrases: perfect measures, strict topologies, Dunford-Pettis Property .

Partially supported by Fondecyt, proyect 89-655 and Dirección de Investigación de la Universidad de Concepción, proyect 20.12.16. 


\section{Perpect Measures}

Let $X$ be a completely regular Hausdorff space, and let $C_{b}(X)$ denote the space of all bounded continuous real-valued functions on $X ; M(X)$ denotes the dual of $\left.\left(C_{b}(X), \|\right\}\right)$ which is, by The Alexandroff Representation's Theorem, the space of all zero set regular, finitely additive set functions on the Baire Algebra $B a^{*}(X)$. Basic references for the theory of measure on topological spaces are on [6]. A countable additive measure $\mu$ on a measurable space $(S, \mathcal{A})$ is said to be perfect, if for every $\mathcal{A}$-measurable function $g: S \longrightarrow \mathbb{R}$, there is a Borel set $B$ in $\mathbb{R}$ such that $B \subset g(S)$ and $|\mu|\left(g^{-1}(B)\right)=|\mu|(S)$. Our principal references about perfect measures are on [4]. If $X$ is a completely regular space, $M_{p}(X)$ denotes the space of all perfect measures defined on the $\sigma$-algebra $B a(X)$ of Baire sets. It is known $M_{\sigma}(X) \supset M_{p}(X) \supset M_{t}(X)$ (for definitions of $M_{\sigma}(X)$, and $M_{t}(X)$ see [6] and, if $X$ is a separable metric space, $M_{p}(X)=M_{t}(X)$ [4].

Perfect measures were first defined by Gnedenko and Kolmogorov in 1949. In 1975 Fremlim proved a very interesting result which reveals the usefulness of perfect measures on arbitrary measurable spaces Fremlim's theorem says that every sequence of measurable scalar functions on a finite perfect measurable space has either a subsequence that converges almost everywhere or it has no measurable pointwise cluster point.

Sentilles [5] defined three topologies on $C_{b}(X)$, socalled strict topologies, denoted by $\beta_{0}, \beta$ and $\beta_{1}$ whose duals are $M_{t}(X), M_{\tau}(X)$ and $M_{\sigma}(X)$ respectively. Following this work, Koumoullis [4] defined another topology on $C_{b}(X)$, denoted by $\beta_{p}$, and showed that $\left(C_{b}(X), \beta_{p}\right)^{\prime}=M_{p}(X)$. The definition of this strict topology is based on some special class of sets, the distinguishable sets of Frolik: a subset $G$ of a completely regular space $Y$ is distinguishable if there is a continuous function $\varphi$ from $Y$ onto a separable metric space such that $G=\varphi^{-1}(\varphi(G))$. We denote by $\mathcal{D}(Y)$ the family of distinguisable set in $Y . \mathcal{D}(Y)$ is a $\varphi$-algebra containing the $\sigma$-algebra of Baire sets. For every $Q \subset \beta X-X$, the spaces $C_{b}(X)$ and $C_{b}(\beta X-Q)$ are isomorphics since $\beta\left(\beta(X-Q)=\beta X\right.$. So the topology $\beta_{0}$ on $C_{b}(\beta X-Q)$, which is defined as the finest locally convex topology agreeing with the compact-open topology on all norm bounded subsets, can be regarded as a topology on $C_{b}(X)$ which is denoted by $\beta_{Q}$. It is defined the strict topology $\beta_{p}$ by $\beta_{p}=\operatorname{Lin}\left\{\beta_{G}: G \in \mathcal{D}(X), G \subset \beta X-X\right\}$. Since $\beta_{1}=\operatorname{Lin}\left\{\beta_{Z}: Z\right.$ in a zero set, $Z \subset \beta X-X\}$ and every zero set is distinguishable, we have $\beta_{1}$ is finer than $\beta_{p}$.

The following results can be found in [4].

Lemma 1. If $\mu \in M_{\sigma}^{+}(X)$, then $\mu$ is perfect if and only if for every continuous function $f$ from $X$ onto a separable metric space $M, f_{*}(\mu) \in M_{t}(M)$, where $f_{*}(\mu)(g)=\mu(g \circ f)$.

Theorem 2. A subset $H$ of $M_{p}(X)$ is $\beta_{p}$-equicontinuous if and only if (a) $H$ is bounded, and (b) for every continuous function $f$ from $X$ onto a separable 
metric space $M$ and every $\epsilon>0$, there is a compact $K, K \subset M$, such that $|\mu|\left(X-f^{-1}(K)\right)<\epsilon$ for all $\mu \in H$.

\section{Perfects Measures and the Dunford-Pettis Property}

We shall say that a Hausdorff locally convex space $E$ has the Dunford-Pettis Property (resp. strict Dunford-Pettis Property) if for any Banach space $F$ and every linear continuous weakly compact operator $T: E \longrightarrow F, T(C)$ is relatively compact (resp. $\left\{T x_{n}\right\}$ is Cauchy) in $F$ for any absolutely convex weakly compact subset $C$ (resp. weak Cauchy sequence $\left.\left\{x_{n}\right\}\right) \in E$.

Let $T$ be a linear operator defined from $C_{b}(X)$ into a Banach $E$ which is norm-continuous' let $m$ denote its associated measure. The following relations will be used: $\|T f\|=\sup \left\{\left|x^{\prime} \circ T(f)\right|:\left\|x^{\prime}\right\| \leq 1\right\}$.

The first theorem of this work gives a characterization of the weakly compact operators defined on $C_{b}(X)$ into a Banach space $E$ which are $\beta_{p}$-continuous.

Theorem 3: Let $T$ be a bounded linear weakly compact operator defined on $C_{b}(X)$ into a Banach space $\mathrm{E}$, and let $m$ be its associated vector measure. The following statements are equivalent:

a) $T$ is $\beta_{p}$-continuous.

b) For every continuous mapping g from $X$ onto a separable metric space $M$, and for every $\epsilon>0$, there exists a compact subset $K_{\epsilon}$ of $M$ such that $\|m\|\left(X \backslash g^{-1}\left(K_{\epsilon}\right)\right)<\epsilon$.

\section{Proof:}

a) $\Rightarrow$ b) Suppose $T$ is $\beta_{p}$-continuous, this implies that the subset $\left\{x^{\prime} \circ m:\left\|x^{\prime}\right\| \leq 1\right\}$ is $\beta_{p}$ - equicontinuous; therefore, for every $\epsilon>0$, and for every continuos map $g$ from $X$ onto a separable metric space $M$, there exists a compact subset $K$ of $M$ such that $\left|x^{\prime} \circ m\right|\left(X \backslash g^{-1}(K)\right)<\epsilon / 2$, for all $x^{\prime},\left\|x^{\prime}\right\| \leq 1$ [4]. The statement folows from the fact that

$$
\|m\|\left(X \backslash g^{-1}(K)\right)=\sup \left\{\left|x^{\prime} \circ m\right|\left(X \backslash g^{-1}(K)\right):\left\|x^{\prime}\right\| \leq 1\right\} .
$$

b) $\Rightarrow$ a) It is enough to prove $H=\left\{x^{\prime} \circ m:\left\|x^{\prime}\right\| \leq 1\right\}$ is bounded, since $\|m\|(X)<$ $\infty$. Also, by the fact $\left|x^{\prime} \circ m\right|(A) \geq\|m\|(A)$, for all $\left\|x^{\prime}\right\| \leq 1$ and for all Baire subset $A$ of $X$, and from $X$ onto a separable metric space $M$, there exists a compact subset $K$ of $M$ such that $\left|x^{\prime} \circ m\right|\left(X \backslash g^{-1}(K)\right)<\epsilon$, for all $x^{\prime},\left\|x^{\prime}\right\| \leq 1$. These two facts and Theorem 2 show $H$ is $\beta_{p}$-equicontinuous. The statement follows from this since $\|T f\|=\sup \left\{\left|x^{\prime} \circ T(f)\right|:\left\|x^{\prime}\right\| \leq 1\right\}$ and $\left\{\left|x^{\prime} \circ T(f)\right|:\left\|x^{\prime}\right\| \leq 1\right\}$ is $\beta_{p}$-equicontinuous. 
Theorem 4. $\left(C_{b}(X), \beta_{p}\right)$ has the strict Dunford-Pettis property.

Prooof: Since $\beta_{p} \geq \beta_{1}$, a linear operator $\beta_{p}$-continuous $T$ is also $\beta_{1}$-continuous.

Let $T$ be a linear $\beta_{1}$-continuous operator of $C_{b}(X)$ to $E$ which is weakly compact, and let $\left\{f_{n}\right\}_{n \in I N}$ be a weakly Cauchy sequence in $C_{b}(X)$. Then $\left\{f_{n}(x)\right\}_{n \in N}$ is Cauchy in $\mathbb{R}$ for each $x \in X$.

On the other hand, the associated vector measure $m$ of $T$ is $\sigma$-additive and then it admits a real-valued Baire control measure $\mu$, that is, given $\epsilon>0$, there exists $\delta>0$ such that for every Baire subset $F$ of $X, \mu(F)<\delta$ implies $\|m\|(F)<\epsilon$.

Now, since $\left\{f_{n}\right\}_{n \in \mathbb{N}}$ converges pointwise in $X$, we have that, by Egoroff's Theorem, there exists a Baire subset $F_{\delta}$ of $X$ such that $\left\{f_{n}\right\}_{n \in N}$ converges uniformly on $X-F_{\delta}$ and $\mu\left(F_{\delta}\right)<\delta$.

Let $n_{0} \in \mathbb{N}$ such that for $n, m \leq n_{0}$

$$
\sup \left\{\left\|f_{n}(x)-f_{m}(x)\right\|: x \in X-F_{\delta}\right\}<\epsilon /(2 M) \text {, where } M=\|m\|(X) .
$$

Thus

$$
\begin{aligned}
\left\|T f_{n}-T f_{m}\right\| & \leq\left\|\int_{X-F_{\delta}}\left(f_{n}-f_{m}\right) d m\right\|+\left\|\int_{F_{\delta}}\left(f_{n}-f_{m}\right) d m\right\| \\
& \leq \sup \left\{\left\|f_{n}(x)-f_{m}(x)\right\|: x \in X-F_{\delta}\right\}\|m\|(X)+L\|m\|\left(F_{\delta}\right)<\epsilon,
\end{aligned}
$$

where $\left\|f_{n}\right\| \leq L$ for all $n \in \mathbb{N}$.

Theorem 5. If $X$ is $\sigma$-compact, then $\left(C_{b}(X), \beta_{p}\right)$ has the Dunford-Pettis property.

Proof: Let $\left\{K_{n}\right\}$ be an increasing sequence of compact subset of $X$ whose union $\cup K_{n}$ in dense in $X$. Denoting by $B_{n}$ the closed unit ball of $\left(C\left(K_{n}\right),\|\|\right)^{\prime}$, we get for any $\mu \in B_{n}, \tilde{\mu} \in M_{p}(X)$ defined by $\tilde{\mu}(f)=\mu\left(f_{\mid K_{n}}\right)$. In fact, first of all observe that $\tilde{\mu} \in M_{\sigma}(X)$ since if $f_{m} \downarrow 0$, then $f_{m} \longrightarrow 0$ uniformly on $K_{n}$ (by Dini's Theorem) which implies $\tilde{\mu}\left(f_{m}\right)=\mu\left(f_{m \mid K_{n}}\right) \longrightarrow 0$. Let $g$ be a continuous function from $X$ onto a separable metric space $M$, and consider the induced map $g_{*}: M_{\sigma}(X) \longrightarrow M_{\sigma}(M)$, defined by $g_{*}(\mu)(B)=\mu\left(g^{-1}(B)\right)$ for all Baire subset of $M$. In order to prove $\tilde{\mu} \in M_{p}(X)$, by Lemma 1 , we have to show $g_{*}(|\tilde{\mu}|) \in M_{t}(M)$. Take a $h_{\alpha} \longrightarrow 0$ in the compact-open topology on the unit ball of $C_{b}(M)$, without lost of generality we assume $h_{\alpha} \geq 0, g_{*}(|\tilde{\mu}|)\left(h_{\alpha}\right)=|\tilde{\mu}|\left(h_{\alpha} \circ g\right) \leq$ $|\mu|\left(\left(h_{\alpha} \circ g\right)_{\mid K_{n}}\right) \longrightarrow 0$ since $h_{\alpha} \longrightarrow 0$ uniformly on the compact subset $g\left(K_{n}\right)$, this says $g_{*}(|\mu|) \in M_{t}(M)$.

Putting $\tilde{B}_{n}=\left\{\tilde{\mu}: \mu \in B_{n}\right\}$ and taking a net $\left\{\tilde{\mu}_{\beta}\right\}$ such that $\mu_{\beta} \longrightarrow \mu \in B_{n} \quad$ (note $B_{n}$ is compact in weak*-topology), i.e. $\mu_{\beta}(f) \longrightarrow$ 
$\mu(f), \forall f \in C\left(K_{n}\right)$. This implies $\tilde{\mu}_{\beta}(f) \longrightarrow \tilde{\mu}(f) \quad \forall f \in C_{b}(X)$ and so $\tilde{B}_{n}$ is compact in $\left(M_{p}(X), \sigma\left(M_{p}(X), C_{b}(X)\right)\right.$.

The vector subspace $B=\cup_{n, m \in N} m \tilde{B}_{n}$ is dense in $\left(M_{p}(X), \sigma\left(M_{p}(X)\right.\right.$, $\left.C_{b}(X)\right)$, since if $f \in C_{b}(X)$ and $f \equiv 0$ on $X$. Therefore, $\left(M_{p}(X), \sigma\left(M_{p}(X)\right.\right.$, $\left.C_{b}(X)\right)$ is $\sigma$-compact. The result now tollows from the fact that $\left(C_{b}(X), \beta_{p}\right)$ possesses the strict Dunford-Pettis property and Theorem 1 [3].

\section{REFERENCES}

[1] Aguayo, J.; Sánchez, J.: Weakly Compact Operators and the Strict Topologies. Bull. Austral. Math. Soc., 39, 1989.

[2] Aguayo, J.; Sánchez, J.: Separable Measures and The Dunford-Pettis Property. Bull. Austral. Math. Soc.. 43, 1991.

[3] Khurana, S.S.: Dunford-Pettis Property. J. Math. Anal. Appl.. 65, 1978.

[4] Koumoullis, G.: Perfect, $\mu$-additive Measures and Strict Topologies. Illinois J. of Math. 26, N³, 1982.

[5] Sentilles, F.: Bounded continuous functions on a completely regular spaces. Trans. Amer. Math. Soc. 168, 1972.

[6] Varadarajan, V.: Measures on topological spaces. Amer. Malh. Soc. Transl. 48, 1965.

Recibido: $\gamma$ Septiembre de 1992.

José Aguayo G. and José Sánchez H.

Departamento de Matemática

Universidad de Concepción

Casilla 3-C, Concepción, Chile 